\title{
Proposta de Atualização e Integração das Disciplinas de Geotecnologia do Curso de Geologia do Instituto de Geociências da USP
}

\author{
Arlei Benedito Macedo (abmacedo@usp.br) e Teodoro Isnard Ribeiro de Almeida \\ Departamento de Geologia Sedimentar e Ambiental - Instituto de Geociências - USP \\ R. do Lago 562, CEP 05508-080, São Paulo, SP, BRA
}

Recebido em 12 de dezembro de 2006; aceito em 01 de fevereiro de 2007

Palavras-chave: Geociências, Geotecnologia, Geoprocessamento, Sensoriamento Remoto.

\section{RESUMO}

A área de conhecimento da Geotecnologia é definida como o estudo e aplicação das técnicas de levantamento e utilização de informações métricas e gráficas para as ciências da Terra. No currículo do curso de Geologia abrange conhecimentos e técnicas de Topografia, Desenho, Cartografia, Geoprocessamento e as técnicas de uso de produtos de Sensoriamento Remoto que se referem à extração de informações métricas e sua representação em mapas, não incluindo a interpretação geológica ou ambiental desses produtos. No atual curso de Geologia do IGc-USP, a Geotecnologia está dispersa em seis disciplinas, das áreas de Topografia, Geoprocessamento e Sensoriamento Remoto, o que ocasiona repetições de partes das ementas, ao mesmo tempo em que outras não podem ser tratadas com a extensão necessária para seu uso eficiente. Propõe-se a instituição de uma nova disciplina, denominada “Geotecnologia para Geologia”, abrangendo os conhecimentos mencionados, exceto os da disciplina Topografia, que deve continuar a ser ministrada na Escola Politécnica, pois sua incorporação exigiria a aquisição de equipamentos e contratação de pessoal especializado, não justificável em face do benefício de sua incorporação ao IGc. Deve ser feito um trabalho de integração entre as equipes da Poli e do IGc para melhor aproveitamento das duas disciplinas. A nova disciplina abrangerá 60 horas-aula e 60 de prática individual (treinamento de programas e projeto piloto).

Keywords: Geoscience, Geotechnology, GIS, Remote Sensing.

\begin{abstract}
The area of Geotechnology is defined as the study and application of techniques of survey and use of metric and graphic information for the Earth Sciences. The Geology course curriculum comprises the knowledge and techniques of Topography, Drafting, Cartography, Geographic Information Systems and Remote Sensing, in those parts referring to extraction of metric information of remote sensing products and their representation in maps, not including their geologic or environmental interpretation. In the present Geology course at IGc-USP, Geotechnology is dispersed among six subjects, in the Topography, Geographic Information Systems and Remote Sensing areas, causing repetitions of parts of the subject matter, and at the same time others can not be treated in the depth needed for their efficient application. Creation of a new subject called "Geotechnology for Geology" is proposed, including the topics mentioned, except those of topography, which should still be taught at the Escola Politécnica, as their inclusion as an IGc course would demand acquisition of equipment and hiring of specialized personnel, which is not justifiable if we consider the cost-benefits of their incorporation by the IGc. Integration of the work developed by the different schools should be undertaken, for better performance on both subjects. The new subject will have 60 class hours and 60 of individual practice (software training and pilot project).
\end{abstract}




\section{INTRODUÇÃO}

A área de conhecimento das Geotecnologias é definida como o estudo e aplicação das técnicas de levantamento e utilização de informações métricas e gráficas para as ciências da Terra. No currículo do curso de Geologia abrange conhecimentos e técnicas de Topografia, Desenho, Cartografia, Geodésia, Geoprocessamento e as técnicas de uso de produtos de Sensoriamento Remoto que se referem à extração de informações métricas e sua apresentação em mapas, não incluindo a interpretação geológica ou ambiental desses produtos. No atual curso de Geologia do IGc-USP, esta área de conhecimento está dispersa em seis disciplinas, abaixo discriminadas, o que ocasiona repetições de partes das ementas, ao mesmo tempo em que outras não podem ser tratadas com a extensão necessária para seu uso eficiente. Algumas dessas disciplinas são atualmente optativas, mas seu conteúdo é fundamental para que o geólogo exerça suas funções eficientemente, tanto nas atividades acadêmicas como profissionais. É o caso das técnicas de Geoprocessamento, de posicionamento por satélite (tratada em Elementos de Geodésia) e do Processamento Digital de Imagens.

Propõe-se a instituição de uma nova disciplina, denominada “Geotecnologia para Geologia”, abrangendo os conhecimentos mencionados, exceto os da disciplina Topografia, que deve continuar a ser ministrada na Escola Politécnica, pois sua incorporação exigiria a aquisição de equipamentos e contratação de pessoal especializado, não justificável em face do benefício de sua incorporação ao IGc. Deve ser feito um trabalho de integração entre as equipes da Poli e do IGc para melhor aproveitamento das duas disciplinas.

\section{PROPOSTA}

A disciplina “Geotecnologia para Geologia” substituirá total ou parcialmente as disciplinas seguintes, como disciplina obrigatória; dependendo dos docentes envolvidos, as disciplinas poderão ser mantidas como optativas, ou substituídas por optativas tratando de tópicos mais avançados:

1. PCC2110 - Desenho para Geologia - 2 créditos;

2. AGG0302 - Elementos de Geodésia - 4 créditos;

3. GSA0487 - Aplicações Geológicas de Geoprocessamento - 4 créditos;

4. GSA0489 - Aplicações de Processamento Digital de Imagens (parcialmente) - 1 crédito;

5. 0440200 - Sensoriamento Remoto e Fotogeologia parcialmente - 3 créditos (estas duas disciplinas devem ser replanejadas, juntando-as numa disciplina de Interpretação de Produtos de Sensoriamento Remoto).

A ementa será como apresentada na Tabela 1.

A disciplina abrangerá aulas teóricas, palestras por especialistas, demonstrações de equipamentos e programas e atividades práticas, compreendendo treinamento no uso de programas de computador e um projeto piloto individual, que consistirá na produção de um SIG geológico e seus produtos cartográficos.

A presente proposta é preliminar, fruto de discussão de docentes e alunos interessados na melhoria do curso de Geologia, tendo sido ligeiramente modificada a partir de sua apresentação no I Seminário do Currículo do Curso de Geologia do Instituto de Geociências da USP. Ela deverá ser submetida a discussão pelos departamentos envolvidos e ser aprovada pela Comissão de Graduação e Congregação.

Tabela 1. Ementa da disciplina proposta "Geotecnologia para Geologia".

\begin{tabular}{|c|c|c|}
\hline Disciplinas atuais & Ementa para Geotecnologia & Horas \\
\hline Desenho para & Noções básicas de desenho em papel e computador & 2 \\
\hline Geologia & Normas e técnicas de desenho para topografia e cartografia básica e geológica & 2 \\
\hline \multirow{2}{*}{ Geodésia } & Posicionamento por satélite & 2 \\
\hline & Projeções cartográficas & 2 \\
\hline \multirow{6}{*}{$\begin{array}{l}\text { Fotogeologia e } \\
\text { Sensoriamento } \\
\text { Remoto }\end{array}$} & Noções de princípios físicos de SR (energia, origem da propagação, comportamento espectral de alvos) & 4 \\
\hline & Sensores e plataformas - fotográficos e digitais & 4 \\
\hline & Processamento de produtos de sensoriamento remoto: pré-processamento geométrico e espectral & 4 \\
\hline & Realces espectrais e espaciais; classificação & 8 \\
\hline & Retificação de fotos e imagens de baixa altitude & 4 \\
\hline & Produção de cartas a partir de fotos e imagens & 4 \\
\hline \multirow{6}{*}{ Geoprocessamento } & Definições, funções, hardware e software para Geoprocessamento e processamento de Sensoriamento Remoto & 8 \\
\hline & Entrada de dados e montagem de SIGs & 8 \\
\hline & Análise em Geoprocessamento & 8 \\
\hline & Total parte teórica & 60 \\
\hline & Prática - treinamento de programa e projeto piloto individual & 60 \\
\hline & Créditos: 4 aula / 4 trabalho & \\
\hline
\end{tabular}




\author{
ANEXOS \\ Ementas das atuais disciplinas conforme Website do IGc-USP, \\ <http://www.igc.usp.br/ensino/graduação/disciplinas>, consultado em 14 out. 2006 \\ Escola Politécnica Engenharia de Transportes \\ Disciplina: PTR0201 - Topografia Geral
}

\section{Créditos Aula: 4 \\ Créditos Trabalho: 0 \\ Carga Horária Estágio: \\ Tipo: Semestral}

\section{Objetivos}

\section{Docente(s) Responsável(eis) \\ Carlos Yukio Suzuki \\ Nicola Pacileo Netto}

\section{Programa Resumido}

\section{Programa}

Conceitos básicos definidos na NBR_13.133.Medições de distâncias e de ângulos. Aplicações da teoria dos erros na Topografia Clássica. - Planimetria: cálculo de azimutes e coordenadas. Nivelamento: barométrico, trigonométrico e geométrico. Taquiometria. Sistema geodésico brasileiro. Projeção UTM: conceitos, transformações e cálculos. GPS (Global Positioning System): estrutura dos satélites e dos sinais GPS. Levantamento subterrâneo: giroscópios e outros instrumentos para obras subterrâneas. Representação do relevo, avaliação de áreas e de volumes, estudos sobre a planta topográfica, locação topográfica, desenho topográfico.

\author{
Avaliação \\ Método \\ Aulas expositivas. Aulas práticas com instrumentos \\ topográficos.

\section{Critério} \\ $\mathrm{A}=2 \mathrm{P}+\mathrm{T}=53$ onde: $\mathrm{P}=$ média das provas sendo $\mathrm{P}=$ \\ $5 \mathrm{~T}=$ média dos trabalhos práticos.

\section{Norma de Recuperação} \\ 01 (uma) prova na primeira semana de aula do semestre \\ seguinte.
}

\section{Bibliografia}

NBR 13133 - Execução de Levantamento Topográfico - Procedimento - 1994. PACILÉO NETTO, NicolaAplicações da Teoria dos Erros, Apostilas - PTR/LTG. CINTRA. Jorge Pimentel, Exercícios de Topografia. CINTRA, Jorge Pimentel, Sistema UTM. Apostila PTR/LTG. BLITZKOW, Denizar, Posicionamento Geodésio por Satélite, Apostila, PTR/LTG, 1995.

\title{
Instituto de Geociências
}

\section{Disciplinas Interdepartamentais do Instituto de Geociências \\ Disciplina: 0440200 - Sensoriamento Remoto e Fotogeologia}

Créditos Aula: 6

Créditos Trabalho: 0

Carga Horária Estágio:

Tipo: Anual

\section{Objetivos}

Fornecer elementos sobre sensoriamento remoto, sistemas sensores, comportamento espectral dos materiais superficiais da Terra, interpretação de fotografias aéreas e imagens; noções sobre processamento digital de imagens.

\section{Docente(s) Responsável(eis)}

Coriolano de Marins e Dias Neto

\section{Programa Resumido}

Fornecer elementos sobre os sistemas sensores, comportamento espectral dos materiais superficiais da Terra, interpretação de fotografias aéreas e imagens, e informações 
sobre os principais programas de sensoriamento remoto.

\section{Programa}

- Sensoriamento remoto - definições e histórico

- Princípios físicos do sensoriamento remoto

- Propriedades espectrais de alvos naturais

- Características dos sistemas sensores

- Princípios de fotointerpretação

- Sistemas imageadores - Landsat, SPoT, Radar

- Elementos de processamento digital de imagens

- Aplicações práticas do sensoriamento remoto em geologia e outros campos das ciências da natureza

Prática: Manuseio de fotografias e imagens.

Exercícios com fotos convencionais e interpretação fotogeológica.

Exercícios com imagens Landsat ou SPoT.

Exercícios com imagens de Radar.

\section{Avaliação}

Método

O curso será desenvolvido com base em aulas teóricas, aulas de campo e exercícios em sala de aula.

\section{Critério}

Provas e relatórios dos trabalhos práticos.

\section{Norma de Recuperação}

Não há recuperação.

\section{Bibliografia}

COLWELL, R.N. (Ed.) Manual of Remote Sensing, 2nd Ed. American Society of Photogrammetry, 2 vols. Falls
Church, 1983.

CURRAN, P.J. Principles of Remote Sensing, Longman Sci \& Tech., Essex, 1985.

SABINS, F.F. Remote Sensing: principles and interpretations, 2nd Ed., W.H. Freeman, San Francisco, 1983.

SIEGAL, B.S. \& GILLESPIE, A.R. Remote Sensing in Geology, John Wiley \& Sons, New York, 1980.

SOARES, P.C. \& FIORI, A.P. Lógica e sistemática na análise e interpretação de fotografias aéreas em Geologia. Not. Geom. Campinas, 18(38):71-104, dezembro, 1976.

RICCI, M. \& PETRI, S. Princípios de aerofotogrametria e interpretação geológica, Companhia Editora Nacional, São Paulo.

CRÓSTA, A.P., Processamento Digital de Imagens de Sensoriamento Remoto, Unicamp, Campinas, 1993, 170 p.

NOVO, E.M.L.M., Sensoriamento Remoto-princípios e aplicações, Edgard Blücher Ltda., São Paulo, 1989, 308 p.

DRURY,S.A., Image Interpretation in Geology, Allen e Unwin, Londres, 1987, $243 \mathrm{p}$.

LILLESAND, T.M. \& KIEFFER, R.W., Remote Sensing and Image Interpretation, John Wiley, Nova York, 1987, $521 \mathrm{p}$.

\section{Instituto de Geociências Geologia Sedimentar e Ambiental Disciplina: GSA0487 - Aplicações Geológicas de Geoprocessamento}

\section{Créditos Aula: 4 \\ Créditos Trabalho: 0 \\ Carga Horária Estágio: \\ Tipo: Semestral}

\section{Objetivos}

Habilitar os alunos a utilizar as técnicas de geoprocessamento para trabalhar em geologia básica e aplicada.

\section{Docente(s) Responsável(eis)}

Arlei Benedito Macedo

\section{Programa Resumido}

Introdução ao geoprocessamento. Programas, equipa- mentos e técnicas. Fontes de dados. Modelos numéricos de terreno. Técnicas de análise espacial. Aplicações em Petrologia, Pedologia, Geohidrologia, Topografia, Mapeamento Geológico, Geomorfologia, Geologia Ambiental, Pesquisa Mineral e Mineração.

\section{Programa}

- Introdução aos sistemas de informação geográfica e as técnicas de geoprocessamento

- Programas de computador para geoprocessamento

- Estruturas e qualidade de dados em geoprocessamento

- Equipamentos e técnicas de entrada, arquivamento e saída de dados

- Fontes de dados geológicos para geoprocessamento: 
mapas, fotos, imagens digitais

- Modelos numéricos de terreno e suas aplicações

- Técnicas de análise espacial aplicáveis aos estudos geológicos

- Aplicações de geoprocessamento em: Petrologia, Pedologia, Hidrogeologia, Topografia, Mapeamento Geológico, Geomorfologia, Geologia Ambiental, Pesquisa Mineral e Mineração

\section{Avaliação}

Método

Provas, exercícios e projeto de geoprocessamento aplicado à geologia.

\section{Critério}

Nota mínima 5,0, média ponderada com peso 4 para provas, 2 para exercícios e 4 para projeto.

\section{Norma de Recuperação}

Trabalho prático: projeto de geoprocessamento, diferente do realizado durante as aulas, entregue antes do início do semestre subseqüente ao da ministração da disciplina.

\section{Bibliografia}

Bibliografia Básica:

ASSAD, E.D. \& SANO, E.E., 1998 - Sistema de Informações Geográficas. Brasília, SPI-Embrapa, 434 p.

BONHAM-CARTER, G.F., 1994 - Geographic Information Systems for Geoscientists: Modelling with GIS. Ottawa, Pergamon, 398 p.

\section{Escola Politécnica - Engenharia de Construção Civil Disciplina: PCC2110 - Desenho para Geologia}

Créditos Aula: 2

Créditos Trabalho: 0

Carga Horária Estágio:

Tipo: Semestral

\section{Objetivos}

Desenvolver habilidades do aluno ligadas à visualização espacial. Capacitá-lo para o uso de ferramentas gráficas para representação e resolução de problemas espaciais.

\section{Docente(s) Responsável(eis)}

Eduardo Toledo Santos

\section{Programa Resumido}

1.Técnicas de esboço;

2. Projeções cotadas;

3. Superfícies topográficas;

4. Desenho geométrico;

5. Introdução ao CAD;

6. Modelamento 3D: arestas, faces, superfícies e sólidos;

7. Geometria Descritiva;

8. Vistas ortográficas principais e auxiliares;

9. Cotagem;

10. Perspectiva isométrica.

\section{Programa}

1.Técnicas de esboço;

2. Projeções cotadas;

3. Superfícies topográficas;

4. Desenho geométrico;
5. Introdução ao CAD;

6. Modelamento 3D: arestas, faces, superfícies e sólidos;

7. Geometria Descritiva;

8. Vistas ortográficas principais e auxiliares;

9. Cotagem;

10. Perspectiva isométrica.

\section{Avaliação}

Método

Autoestudo, Aula expositiva, Atividades práticas individuais e em grupo.

\section{Critério}

MF $=($ Prova1 + Prova2 + Média de Exercícios $) / 3$

\section{Norma de Recuperação}

Critério de Aprovação: $\mathrm{A}=(\mathrm{R}+\mathrm{MF}) / 2>=5 \mathrm{R}=$ nota da prova de recuperação $\mathrm{MF}=$ média final antes da recuperação

\section{Bibliografia}

EQUIPE DE PROFESSORES. Apostilas de PCC2100. Escola Politécnica, 2002.EQUIPE DE PROFESSORES. Apostilas de PCC2101. Escola Politécnica, 2002.LANDI, F. et al., Desenhov. 1 e3, São Paulo: Escola Politécnica, 1984.MACHADO,A. Desenho na Engenharia e Arquitetura v.1, 3. ed. São Paulo: A. Machado, 1980.255p·MARMO, C.Curso de Desenho v.1 e 2., 1. ed. São Paulo: Moderna, 1964. GIESECK, et al., Comunicação Gráfica Moderna, 1. ed. Porto Alegre: Bookman, 2002.BENTLEY SYSTEMS, Microstation User's Guide.'MACHADO, A. Geometria Descritiva, 24a Edição. São Paulo: McGraw-Hill, 1974. 


\section{Instituto de Geociências - Geologia Sedimentar e Ambiental Disciplina: GSA0489 - Aplicações de Processamento Digital de Imagens}

\author{
Créditos Aula: 4 \\ Créditos Trabalho: 0 \\ Carga Horária Estágio: \\ Tipo: Semestral
}

\section{Objetivos}

Introduzir aos alunos os principais métodos de processamento digital de imagens (PDI) no visível e infravermelho. Efetuar treinamento em um ou mais sistemas. Gerar condições para que os alunos desenvolvam um trabalho de aplicação de PDI.

\section{Docente(s) Responsável(eis)}

Teodoro Isnard Ribeiro de Almeida

\section{Programa Resumido}

Principais técnicas de processamento digital de imagens no visível e infravermelho. Manejo de processador de imagens. Uso de imagens orbitais multi-espectrais.

\section{Programa}

Revisão de Princípios Físicos do Sensoriamento Remoto; revisão das características de produtos de sensoriamento remoto no visível e infravermelho. A estrutura da imagem. Manipulação de histogramas de Dns; Prática. A cor no pdi; composições coloridas; Prática. Transformação HSI; Prática. Filtragens; Prática. Fatiamento e aplicação da Falsa - cor;
Prática. O reconhecimento automático de padrões: classificações. Máximo Uso semelhança; Prática. Paralelepípedo; Prática. Definição de área e objetivos para o projeto. Desenvolvimento do projeto sob orientação. Apresentação dos resultados em Sessão Painel.

\section{Avaliação}

Método

Aulas expositivas. Aulas práticas no Laboratório. Desenvolvimento de projeto sob orientação.

\section{Critério}

Trabalho final e desempenho na apresentação do painel.

\author{
Norma de Recuperação \\ Conforme a Res. CoG 3583, de 29.09.89.
}

\section{Bibliografia}

CROSTA, A.P. (1993). Processamento Digital de Imagens de Sensoriamento Remoto. UNICAMP. 170 p.

LILLESAND,T.M. \& KIEFER, R.W. (1994). Remote Sensing and Image Interpretation. John Wiley \& Sens. New York. $750 \mathrm{p}$.

DRURY, S.A. (1993). Image Interpretation in Geology. Chapman \& Hall Londres. 283 p.

\section{Geofísica - Instituto de Astronomia, Geofísica e Ciências Atmosféricas Disciplina: AGG0302 - Elementos de Geodésia}

Créditos Aula: 4

Créditos Trabalho: 0

Carga Horária Estágio:

Tipo: Semestral

\section{Objetivos}

Fornecer os conhecimentos básicos sobre a forma e a dimensão da Terra. Estudar o posicionamento geodésico usando o GPS.

\section{Docente(s) Responsável(eis)}

Nelsi Cogo de Sa

\section{Programa Resumido}

Aspectos históricos e conceitos. Sistemas de coordenadas. Modelos terrestres. Coordenadas geodésicas. Posicionamento geodésico por satélite. Redes de nivelamento. O campo de gravidade da Terra e o seu papel no conhecimento da estrutura terrestre. Projeções cartográficas.

\section{Programa}

Aspectos históricos e conceitos. Sistemas de coordenadas. Modelos terrestres. Coordenadas geodésicas. Posicionamento geodésico por satélite. Redes de nivelamento. O campo de gravidade da Terra e o seu papel 
no conhecimento da estrutura terrestre. Projeções cartográficas.

\section{Avaliação}

Método

Aulas expositivas e exercícios.

\section{Critério}

Provas e trabalhos.

Norma de Recuperação

Uma prova abordando todo o conteúdo da disciplina, a ser dada uma semana antes do início das aulas do semestre seguinte.

\section{Bibliografia}

SÁ, N.C. de - "Elementos de Geodésia”, Texto de aulas, IAG/USP, São Paulo, 1990.

TORGE, W. - “Geodesy”. Walter de Gruyter, New York, 1980.

VANICEK, P. \& KRAKIWSKY, B. - “Geodesy: The Concepts”, Elsevier, New York, 1982.

SÁ, N.C. de, 2003 - “GPS: fundamentos e aplicações”. Texto de Aulas. IAG/USP, São Paulo. 